\title{
Acknowledgment to Reviewers of Games in 2019
}

\section{Games Editorial Office}

MDPI, St. Alban-Anlage 66, 4052 Basel, Switzerland

Published: 26 January 2020

The editorial team greatly appreciates the reviewers who have dedicated their considerable time and expertise to the journal's rigorous editorial process over the past 12 months, regardless of whether the papers are finally published or not. In 2019, a total of 49 papers were published in the journal, with a median time to first decision of 30.72 days and a median time from submission to publication of 73 days. The editors would like to express their sincere gratitude to the following reviewers for their generous contribution in 2019:

Abdalzaher, Mohamed S.

Adriani, Fabrizio

Akhmetzhanov, Andrei R.

Aleksandr, Alekseev

Alm, James

Alt, Marius

Alvarez-Bermejo, Jose Antonio

Ansink, Erik

Aragones, Enriqueta

Archetti, Marco

Arieli, Itai

Attanasi, Giuseppe

Attanasi, Giusppe

Aurell, Alexander

Badicu, Georgian

Bartholomae, Florian

Béal, Sylvain

Bilancini, Ennio

Biondo, Alessio Emanuele

Boonen, Tim J.

Brownback, Andy

Bruttel, Lisa

Buchen, Clemens

Cartwright, Edward

Casajus, André

Castillo, Geoffrey

Cavalli, Fausto

Chessa, Michela

Coatney, Kalyn

Cunningham, David E.
Dahm, Matthias

Dalhaus, Tobias

Danese, Giuseppe

DE MARCO, Giuseppe

Deer, Lachlan

Delaney, Jason

Di, Sharon

Dimant, Eugen

Doruk, Iris

Dürsch, Peter

Ellis, Andrew

Erovenko, Igor

Espinosa, Maria Paz

FARVAQUE, Etienne

Fernández-Revelles, Andrés B.

Filson, Darren

Fochmann, Martin

Fooken, Jonas

Frąckiewicz, Piotr

$\mathrm{Fu}$, Jingcheng

Fujita, Toshiyuki

Galliera, Arianna

Gangl, Katharina

Ganzfried, Sam

Ghidoni, Riccardo

Goff, Sandra

Gómez-Miñambres, Joaquín

Gonzalez-Ramirez, María Jimena

Gratton, Gabriele

Grimshaw, Shaun B 
$\mathrm{Gu}$, Junjian

Gueron, Yves

Gürerk, Özgür

Hanappi, Hardy

Hariskos, Wasilios

Harre, Michael

Harrison, Glenn

Hartman, Bruce

Hedges, Jules

Heine, Florian

Heintz, Christophe

Hilbe, Christian

Hochman, Guy

Hod, Shahar

Hoffer, Adam

Holzman, Ron

Howe, Lance

Hoyer, Britta

Huttegger, Simon

Ikerionwu, Charles

Jaffe, Sonia

Jajac, Nikša

Javarone, Marco Alberto

Javarone, Marco Alberto

Jin, Haiming

Johnson, David

Jung, Sungdo

Kawamura, Kohei

Kirstein, Roland

Klazar, Stanislav

Koch, Alexander

Kölle, Felix

Kolokoltsov, Vassili

Koloniari, Georgia

Kramer, Berber

Krockow, Eva

Kroumi, Dhaker

Kupferman, Orna

Kurokawa, Shun

Kurschilgen, Michael

Lanotte, Ruggero

Lee, Jinkwon

Leland, Jonathan
Louis, Philippos

Mantovani, Marco

Manzoni, Elena

Marini, Marco

Marrouch, Walid

Massey, James

Meloni, Sandro

Millner, Edward

Molinero, Xavier

Munger, Michael C.

Musau, Andrew

Naumann, Elias

Nax, Heinrich H.

Nicolò, Antonio

Nielsen, Kirby

Norman, Thomas W. L.

O’Brien, Michael J.

Okada, Isamu

Oncioiu, Ionica

Otjes, Simon

Owen, Guillermo

Pancotto, Francesca

Pawlowitsch, Christina

Pieperhoff, Martina

Pin, Paolo

Podviezko, Askoldas

Prummer, Anja

Puppe, Clemens

Rass, Stefan

Reeck, Crystal

Rettieva, Anna

Robertson, Matthew

Rosa Garcia, Alfonso

Samu, Flóra

SCARTASCINI, CARLOS

Schneider, Frédéric

Schurr, Amos

Schwanebeck, Benjamin

Schwieren, Christiane

Sefton, Martin

Serena, Marco

Shan, Xiaojun (Gene)

Silva, Rui 
Sirghi, Nicoleta

Sopher, Barry

Spraggon, John

Stashko, Allison

Stoddard, Brock

Swope, Kurtis J.

Te Velde, Vera

Tohme, Fernando

Tröger, Thomas

Tyson, Scott

Ueda, Suguru

Van Basshuysen, Philippe

Van Veldhuizen, Roel
Vesperoni, Alberto

Veszteg, Róbert F.

Veszteg, Róbert Ferenc

Vigors, Belinda

Vlassopoulos, Michael

Vyrastekova, Jana

Wang, Chengsi

Yamarik, Steven

Yang, Yongjie

Ye, Min

Zelizer, Adam

Zusai, Dai

(C) 2020 by the author. Licensee MDPI, Basel, Switzerland. This article is an open access article distributed under the terms and conditions of the Creative Commons Attribution (CC BY) license (http://creativecommons.org/licenses/by/4.0/). 